\title{
Saccadic Eye Movements as a Marker of Mental Disorders
}

\author{
F. JAGLA ${ }^{1}$ \\ ${ }^{1}$ Laboratory of Cognitive Neuroscience, Institute of Normal and Pathological Physiology, Slovak \\ Academy of Sciences, Bratislava, Slovakia
}

Received June 13, 2016

Accepted September 5, 2016

\section{Summary}

It is accepted that the formulation of the motor program in the brain is not only the perceptual and motor function but also the cognitive one. Therefore it is not surprising that the execution of saccadic eye movements can by substantially affected be the ongoing mental activity of a given person. Not only the distribution of attention, but also the focusing the attention may influence the main gain of saccades, their accuracy. Patients suffering from mental disorders have strongly engaged their attention focused at their mental processes. The nature of their problems may be linked to perceptual and/or analytical processing. Such so-called mental set may significantly affect their oculomotor activity in the course of their saccadic eye movement examinations. This short comment points out not only to the influence of the contextually guided and generated saccadic eye movements upon their accuracy but also to the distribution and focusing the attention. The effect of the functional brain asymmetry upon the visually generated saccades and the possible effect of biologically active substances upon the voluntary generated saccades are briefly mentioned. All these influences should be taken into account when planning the saccadic eye movement task. It may be concluded that the repetition of the same oculomotor task in a given person has to be introduced. This may help to follow the effect of complex therapy namely.

\section{Key words}

Saccades - Accuracy of saccades - Attention - Hemispheric activation - Mental set

\section{Corresponding author}

F. Jagla, Institute of Normal and Pathological Physiology, Slovak Academy of Sciences, Sienkiewiczova 1, 81371 Bratislava, Slovakia. E-mail: fedor.jagla@savba.sk

\section{Introduction}

The visual-oculomotor integration represents the most subtle as well as the most precise kind of sensorimotor integration. The study of this integration offers the possibility to analyse its "peripheral" (oculomotor) and "central" (EEG) correlates completed with the subjective statements concerning the particular examination. The mechanisms of sensorimotor integration are affected also by the fronto-parietal reciprocal connections forming rather largely segregated circuits. They can be "specialized" for several specific aspects as e.g. the motivation, memory, long-term plans of a subject and others (Rizzolatti and Lupino 2001). Moreover, the fronto-parietal cortico-cortical connections via thalamus are very probably engaged also in some non-specific supramodal influences. We have shown this with modulation of frontal somatosensory evoked potentials and parietal saccadic eye movement related potentials by various cognitive demands (Jergelová et al. 1996, Jagla et al. 1999). It is quite clear that such mechanisms may be affected in patients suffering from various mental disorders quite strongly.

As it is known, as early as 1908 Allen R. Diefendorf and Raymond Dodge published an article in Brain entitled "An Experimental Study of the Ocular Reactions of the Insane from Photographic Records". Their article took the initiative for following studies relating description of the changes in the oculomotor activity of psychiatric patients in order to assess their possible use as a one of the additional diagnostic criterion for a given disorder. The use of oculomotor tests in psychiatry steadily grows since the work of Holzman 
et al. (1973). Ten years after the Holzman group can publish even a review of similar studies (Lipton et al. 1983). Over the last 40 years the saccadic eye movements became as an important area of oculomotor research in psychiatric disorders. In the last years some their very special characteristics are analyzed as a possible endophenotype of a particular disorder.

Saccadic eye movements (SEM) are a short and very rapid eye movement that can be voluntary or reflexive. They are for capturing the image of interest then projecting it onto the most sensitive part of the retina, into the fovea centralis. The patients with mental disorders show irregularities in the process of sensorimotor integration of course of SEM patterns. As the diagnostic tools following three types of saccades are used: a) The visually guided SEM (or pro- or reflexive SEM; Pierrot-Deseilligny et al. 1991a) are reflexive eye movements toward the randomly appearing visual stimulus in the visual field, b) The so-called antisaccades (O’Driscoll et al. 1995) which means instead of looking at the target when it suddenly appears in the visual field the subjects are instructed to look quickly in the opposite direction and trigger a voluntary saccade of equal amplitude and c) The memory guided saccades (PierrotDeseilligny et al. 1991b). The subject is fixating the central visual target and another visual stimulus is presented for a short time in the periphery. After switching the central fixation point of the subject makes a saccade toward the remembered position of the peripheral visual stimulus. In this short review the visually guided saccades are taking into account.

In healthy people the visually guided SEM are in $95 \%$ accurate. The rest may overshoot or undershoot the desired landing position and the saccade is followed by a corrective one. This is the reason why this comment emphasizes the saccadic accuracy as a main saccadic parameter to be taken into account. In is well known that the other saccadic parameters change with aging. SEM latency is increasing with increasing age (Warabi et al. 1984). With increasing age a strong decrease of peak velocity with a simultaneous slight increase of SEM duration was found (Carter et al. 1983). Also the decrease of the SEM amplitude with increasing age was described (Irving et al. 2006) but it does not mean the change of the accuracy.

The programming of saccadic eye movements (SEM) is a result of complex perceptual, volitional and cognitive processes. There was repeatedly demonstrated that programming and execution of saccades are influenced by a wide range of cognitive processes, including those involved in attention, working memory, learning, long term memory and decision making. The analysis of SEM in healthy volunteers provide the suitable opportunity to study the question how the brain integrates the overall visual, acoustic, and somestetic information and the internally generated cognitive demands in the common every day's human behavior. The cortical and subcortical mechanisms involved in the control of the SEM were at some length even described (Sweeney et al. 2007, McDowell et al. 2008). Quite a lot of studies list several abnormalities in the SEM in a variety of psychiatric and neurological disorders. The reality is that there exists a noticeable variability in saccadic oculomotion not only in a particular disorder but across the all categories of disorders as well. The same conclusion stated Gooding and Basso (2008) who pointed to the fact that it may be instructive to administer multiple permutations of the same oculomotor task in the same study.

Concerning the oculomotor activity there are at least two very important issues which should be kept in mind with psychiatric patients in particular. Sensory and motor information conveyed in the central nervous system are being decoded and transformed during their processing at various levels of the central hierarchy. Over the last decades evidence has been accumulated that there exist some rather non-specific neuronal circuits of the human brain which take part in modulation of the information processing with respect to the more general CNS activation level. Among such non-specific influences upon the brain oculomotor activity the focusing of the attention, mental set, or mental imagination may be mentioned (Jagla et al. 1999, Jergelova et al. 1996, Jeannerod 1994).

All three above mental activities can be active/passive in varying degrees in patients with mental disorders and can significantly affect their oculomotor activity. As for the mental set influence the very illustrative are recordings of the saccadic eye movements made by Yarbus (1967) in a healthy subject when viewing the famous image „Un unexpected visitor“ painted by I. E. Repin. The subject examined the reproduction with both eyes under following instructions: 1) Free examination of the picture; 2) estimate the material circumstances of the family in the picture; 3) give the ages of the people; 4) surmise what the family had been doing before the arrival of the unexpected visitor; 5) remember the clothes worn by the people; 
6) remember the position of the people and objects in the room; 7) estimate how long the unexpected visitor had been away from the family. Seven different instructions what does the subject should inspect in the picture and as the result seven different scanning strategies could be seen. The effect of the mental set - that is contextually guided and internally generated visual scanning of the given picture by saccadic eye movements - is very impressive. As Lévy-Schoen et al. (1989) have pointed out the theories of visuomotor mechanisms must take into account not only the classical 'retinal error' input signal, but also perceptual and decisional factors as well as taskspecific strategies adopted by the subjects. In a simplex experimental model situation Dyckman et al. (2007) demonstrated that the saccade-related brain activity is affected by context and controlled by different networks of cortical and subcortical regions. Therefore, it is not surprising that Hauert (1986) has formulated the hypothesis that the motor function is a cognitive function also. Between setting the purpose of movement and its actual execution, integration takes place between motivational, perceptual, and previously overlearned motor components. This all together should be regarded as the preparation of a motor program (de Jong et al. 1999).

Very closely related to the mental set is the type of the hemispheric activation induced by the easily verbalized and/or non-verbalized scenes of on-going mental images of subjects. The higher saccadic accuracy and the shorter SEM preparation time was found with suspected higher right hemisphere activation in the pronounced right-handers, that is with saccades directed to the left as compared to their rightward direction. This finding supports the presupposed existence of the nonspecific information processing within the specific visual and visual-oculomotor neuronal loops while distracting the attention from visual stimuli by on-going mental activity (Jagla et al. 1997).

The attention is an another important factor affecting the various parameters of the SEM. Corbetta et al. (1998) demonstrated that the attention and eye movement processes are not only functionally related, as originally suggested by psychological studies, but also share functional anatomical areas in the human brain. Shepherd et al. (1986) have shown that while it is possible to perform the shift of the attention focusing without making corresponding eye movements, it is not possible to make an eye movement (in the absence of peripheral stimulation) without making a corresponding shift in the focus of attention. Attention is the very important factor affecting the latency and accuracy of saccades. The latency to plan and execute the most naturally occurring saccades (10-15, Bahill et al. 1975) when a healthy subject jumps from one visual target to another is usually about $200 \mathrm{~ms}$ (Carpenter 1988). The actual movement takes 30-60 ms. The saccadic durations are approximately linearly related to their angular displacements from $1^{\circ}$ to $50^{\circ}$ (Yarbus 1967). Their angular velocity is greater with larger saccades of longer duration (Boghen et al. 1974) and may reach 500-600 per second. Saccadic velocity is slower in CNS pathologies (Baloh et al. 1975). The accuracy of saccades as also their velocities became variable not only in CNS pathologies but also when the subject is tired (Bahill et al. 1981).

In addition, the accuracy of horizontal saccades depends upon the functional brain asymmetry also and of the vertical saccades on their upward and downward direction (Jagla et al. 1992). The more accurate saccades are to the left in right-handers and to the right in lefthanders and when directed upwards (Jagla and Zikmund 1981). Another factor should also be considered is the dependence of the saccadic amplitude upon the general shape of the visual stimulus (Over et al. 2007). Taking into account all the above factors we studied the timing of programming and execution of the horizontal saccades by means of the parietal recorded SEMRPs (Armington 1978, Jagla and Zikmund 1994, Jagla et al. 2007, Marton et al. 1983, Thickbroom and Mastaglia 1986). The SEMRPs were recorded also when the saccades were directed to the right or left visual hemifield whereas the visual attention was focused to the given hemi field or to the contralateral one. The longer duration of the premotion positivity and the increased latency of the maximal recruitment of the oculomotor muscle units at the onset of the saccade (the so-called spike potential SP) were found when the saccadic eye movement was elicited by a target suddenly appearing in the not attended visual hemifield comparing to its appearance in the attended one (Jagla et al. 1994). The left/right differences due to the functional brain asymmetry are well-preserved in healthy subjects but they could be lost in patients suffering from certain mental problems (depressive behavior, panic disorder, etc.) (Jagla et al. 2002, 2003).

Moreover, the on-going mental activity during the oculomotor task is the very good example of the effect of divided attention upon the programming the saccadic eye movements. In our another study the 
subjects were asked to catch as fast and accurate as possible the visual target appearing suddenly at the $10^{\circ}$ distance from the central fixation point. Following this part of examination they were asked to repeat this but to perform during the oculomotor test the mental arithmetic task and consecutively the mental imagery task. The shortest duration of the premotion positivity and of the SP latency were registered during the basal condition. The longer values were found with mental arithmetic task and longest ones with mental imagery task. The same is true as for the number of corrective saccades due to the decreased accuracy of saccades at concurrent visual and mental activities (Jagla et al. 1999). In all conditions the time of the first cortical encoding the novel visual information was not affected which confirm the fact that there were no problems with visual perception as such. In both the above experimental studies, that is with focused/non-focused and distributed/non distributed attention the relation to the functional brain asymmetry was preserved. Our results concerning the accuracy of the saccadic eye movements when distracting the attention from visual stimuli support the idea that within the specific visual and visual-oculomotor neuronal loops some non-specific information are processed which probably requires a certain engagement of working memory.

Apart from the analyzing the results of the antisaccadic task (for a more comprehensive review see Hutton 2008) the promising tool in the study of pathophysiological mechanisms involved in various mental disorders is the memory-guided saccade task (Pierrot-Desseiligny et al. 1991b). The memory guided saccades are voluntary movements of eyes and not a reflexive towards a new visual stimulus. It is quite understandable that in this case the more interesting correlate will be their accuracy, respectively changes in their accuracy because they are in principle less accurate than visually guided saccades. Visually guided saccades are in $95 \%$ accurate in general, the memory guided saccades can be accurate in $70 \%$ or less. The inaccuracy of the visually guided saccades does not exceed $0.5^{\circ}$ when the saccade is made between two visual target separated by $10^{\circ}$. Usually only one corrective saccade is enough to correct the inaccuracy and the latency of correction is affected also by the functional brain asymmetry (Jagla and Zikmund 1981). The inaccuracy of memory guided saccades in the same experimental condition reach $1.2-1.5^{\circ}$ and is frequently corrected by more than one corrective eye movement. In such a case the latency of corrective saccades is not affected by the functional brain asymmetry (Cimrova et al. 2011). When recording the memory guided saccades and the experimental task demands patients to direct the glance to different regions in the visual field the results may help to assess his/her spatial orientation, spatial memory and attention functions. The medication of patients is very important in this case. We were able to document that even the single consumption of "physiologically" natural substances are able to influence significantly the accuracy of the memory guided saccades and the number of corrective saccades as well (Cimrova et al. 2011).

\section{Conclusions}

The majority of everyday visual processing requires 3-4 saccades per second (Findlay and Gilchrist 2003) and their amplitudes may vary from a few degrees up to $130 \mathrm{deg}$ of arc (Land 2004). It was demonstrated that the visual target selection is predominantly controlled by the task demands required from the subject (Findlay 1997). In relation to persons with mental disorders the task demands may have significantly lower priority than the internal world of a given patient as opposed to a higher priority in case of healthy volunteers (Foulsham and Underwood 2008). Moreover, emotional content and interindividual difference may significantly affect the eye movement behavior as well (Calvo and Avero 2005, Wong et al. 2005). In healthy humans the brain functions as receiving, processing, storing information and creating proper answers lead to functional and structural changes within the brain described as the neuroplasticity. It enables subjects to adapt their behavior according to external and internal conditions. Dysregulation of plasticity is supposed to play a role in a variety of neuropsychiatric disorders (Drewets 2000, Moghaddam and Homayoun 2008, Pittenger and Duman 2008). Such dysregulation can explain also the disordered visualoculomotor integration in persons suffering from various mental problems. It can be also taken as an good argument why the particular oculomotor finding cannot be assumed as specific for a given mental disorder. Moreover, up to now there is no knowledge on how the specific neuroplasticity changes as such look like, on one hand, and the fact that the attentional and memory influences can be hardly separately assessed, on the second hand. The disordered brain functions are the result of many complex processes and without the functioning neuroplasticity the disordered brain functions and their 
regulatory mechanisms could be never regained. The methodological variability as also the conflicting results in the literature point a need to improve the experimental paradigms.

Saccadic eye movement changes appear to be involved in the psychiatric disorders and may be considered a possible marker of some disorders. It may be supposed that the changes in the saccadic behavior as well as in its EEG correlates may help well to follow up the course of the complex therapy. Similar point of view was formulated by Trillenberg et al. (2004) and Reilly et al. (2008). And as pointed above it is really demanding to administer multiple permutations of the same saccadic oculomotor task in the same study with the same subject. And only then such parameter as an improvement of the oculomotor behavior may be taken as a possible additional characteristic of the improvement of the actual health status of a given patient. To find some general and common characteristics of the particular intraindividual improvements is the task for future research in the area of the saccadic eye movement behavior characteristics in relation to mental disorders.

\section{Conflict of Interest}

There is no conflict of interest.

\section{Acknowledgements}

This work was partially supported by the VEGA Grants 2/0093/14, 2/0080/13 and APVV-14-0840.

\section{References}

ARMIGNTON JC: Potentials that precede small saccades. In: Visual Psychophysics and Physiology. ARMINGTON JC (ed.), Academic Press, New York, 1978, pp 363-372.

BAHILL AT, ADLER D, STARK L: Most naturally occurring human saccades have magnitudes of 15 degrees or less. Invest Ophthalmol Vis Sci 15: 468-469, 1975.

BAHILL AT, BROCKENBROUGH A, TROOST BT: Variability and development of a normative data base for saccadic eye movements. Invest Ophthalmol Vis Sci 21: 116-125, 1981.

BALOH RW, KONRAD HR, SILLS AW, HONRUBIA V: The saccade velocity test. Neurology 25: 1071-1076, 1975.

BOGHEN D, TROOST BT, DAROFF RB, DELL'OSSO LF, BIRKETT JE: Velocity characteristics of normal human saccades. Invest Ophthalmol Vis Sci 13: 619-623, 1974.

CALVO MG, AVERO P: Time course of attentional bias to emotional scenes in anxiety: Gaze direction and duration. Cogn Emot 19: 433-451, 2005.

CARPENTER RHS: Movements of the Eyes. Pion, London, 1988, 593 p.

CARTER JE, OBLER L, WOODWARD S, ALBERT ML: The effect of increasing age on the latency for saccadic eye movements. J Gerontol 38: 318-320, 1983.

CIMROVÁ B, BUDÁČ S, MELICHEROVÁ U, JERGELOVÁ M, JAGLA F: Electrophysiological evidence of the effect of natural polyphenols upon the human higher brain functions. Neuroendocrinol Lett 32: 464-468, 2011.

CORBETTA M, AKBUDAK E, CONTURO TE, SNYDER AZ, OLLINGER JM, DRURY HA, LINENWEBER ML, PETERSEN SE, RAICHLE ME, VAN ESSEN DC, SHULMAN GL: A common network of functional areas for attention and eye movement. Neuron 21: 761-773, 1998.

DE JONG BM, WILLEMSEN ATM, PAANS AMJ: Brain activation related to change between bimanual motor programs. Neuroimage 9: 290-297, 1999.

DREWETS W: Neuroimaging studies of mood disorders. Biol Psychiat 48: 813-829, 2000.

DYCKMAN KA, CAMCHONG J, CLEMENTZ BA, MCDOWELL JE: An effect of context on seccade-related behavior and brain activity. Neuroimage 36: 774-784, 2007.

FINDLAY JM: Saccade target selection in everyday life. Vision Res 37: 617-631, 1997.

FINDLAY JM, GILCHRIST ID: Active Vision: The Psychology of Looking and Seeing. Oxford University Press, Oxford, 2003, $240 \mathrm{p}$.

FOULSHAM T, UNDERWOOD G: What can saliency models predict about eye movements? Spatial and sequential aspects of fixations during encoding and recognition. $J$ Vis 8: 1-17, 2008. doi:10.1167/8.2.6.

GOODING DC, BASSO MA: The tell-tale tasks: A review of saccadic research in psychiatric patient populations. Brain Cogn 68: 371-390, 2008. 
HAUERT CA: The relationship between motor function and cognition in the developmental perspective. Ital J Neurol Sci Suppl 5: 101-107, 1986.

HOLZMAN PS, PROCTOR LR, HUGHES DW: Eye-tracking patterns in schizophrenia. Science 181: 179-181, 1973.

HUTTON SB: Cognitive control of saccadic eye movements. Brain Cogn 68: 327-340, 2008.

IRVING EL, STEINBACH MJ, LILLAKAS L, BABU RJ, HUTCHINGS N: Horizontal saccade dynamics across the human life span. Invest Ophthalmol Vis Sci 47: 2478-2484, 2006.

JAGLA F, ZIKMUND V: Saccadic eye movements and brain hemispheric dominance (in Slovak). Cs Oftalmol 37: 132-137, 1981.

JAGLA F, ZIKMUND V: Differences in eye movement related potentials with visually triggered horizontal and vertical saccades. In: Visual and Oculomotor Functions, Advances in Eye Movement Research. D'YDEWALLE G, VAN RENSBERGEN J (eds), North Holland, Amsterdam, 1994, pp 19-30.

JAGLA F, ZIKMUND V, MASHONKINA TR, YAKIMOFF NA: The accuracy of saccadic eye movements depends upon their horizontal and vertical direction (in Slovak). Bratisl Lek Listy 93: 287-290, 1992.

JAGLA F, ZIKMUND V, FINDLAY JM: The effect of visual attention upon programming of saccadic eye movements. Studia Psychol 36: 350-353, 1994.

JAGLA F, JERGELOVÁ M, ZIKMUND V: Prefrontal correlates of directing the human gaze. Studia Psychol 39: $285-$ $287,1997$.

JAGLA F, JERGELOVA M, ZIKMUND V: Saccadic evoked potentials corresponding to different types of hemispheric activation. Homeost Health Dis 39: 244-246, 1999.

JAGLA F, KUKUMBERG P, JERGELOVÁ M: Central and peripheral oculomotor manifestations in panic disorder. In: Neurophysiology and Behavioural Intervention in Psychosomatics, Stress Disorders and Health Promotion. GOLDWURM GF, COLOMBO F, MASARAKI S (eds), CIANS, Milano, 2002, pp 171-176.

JAGLA F, JERGELOVÁ M, CIRNECI D, KUKUMBERG P: Oculomotor and evoked potential changes in panic and depressive patients. Homeost Health Dis 42: 145-147, 2003.

JAGLA F, JERGELOVÁ M, RIEČANSKÝ I: Saccadic eye movement related potentials. Physiol Res 56: 707-713, 2007.

JEANNEROD M: The representing brain: Neural correlates of motor intention and imagery. Behav Brain Sci 17: 187245, 1994.

JERGELOVÁ M, JAGLA F, ZIKMUND V: Modulation of sensorimotor integrations by voluntary motor acts and their mental representation: Electrophysiological manifestations. Homeost Health Dis 37: 272-273, 1996.

LAND MF: The coordination of rotations of the eyes, head and trunk in saccadic turns produced in natural situations. Exp Brain Res 159: 151-160, 2004.

LÉVY-SCHOEN A, COËFFÉ C, JACOBS AM: Sensory factors are insufficient to define the ocular saccade goal in complex visual fields. Brain Behav Evol 33: 80-84, 1989.

LIPTON RB, LEVY DL, HOLZMAN PS, LEVIN S: Eye movement dysfunctions in psychiatric patients: A review. Schizophr Bull 9: 13-32, 1983.

MARTON M, SZIRTES J, DONAUER N: A comparative study of averaged labda potentials in man and monkey. In: Psychophysiology 1980, Memory, Motivation and Event-Related Potentials in Mental Operations. SINZ R, ROSENZWEIG MR (eds), VEB Gustav Fischer Verlag, Jena, 1983, pp 379-389.

MCDOWELL JE, DYCKMAN KA, AUSTIN BP, CLEMENTZ BA: Neurophysiology and neuroanatomy of reflexive and volitional saccades: Evidence from studies of humans. Brain Cogn 68: 255-270, 2008.

MOGHADDAM B, HOMAYOUN H: Divergent plasticity of prefrontal cortex networks. Neuropsychopharmacology 33: 42-55, 2008.

O'DRISCOLL G, ALPERT N, MATHYSSE S, LEVY S, RAUCH S, HOLZMANN P: Functional neuroanatomy of antisaccade eye movements investigated with positron emission tomography. Proc Natl Acad Sci USA 92: 925 929, 1995.

OVER EAB, HOOGE ITC, VLASKAMP BNS, ERKELENS CJ: Coarse-to-fine eye movement strategy in visual search. Vision Res 47: 2272-2280, 2007.

PIERROT-DESEILLIGNY C, RIVAUD S, GAYMARD B, AGID Y: Cortical control of reflexive visually-guided saccades. Brain 114: 1473-1485, 1991a. 
PIERROT-DESSEILIGNY C, RIVAUD S, GAYMARD B, AGID Y: Cortical control of memory guided saccades in man. Exp Brain Res 83: 607-607, 1991 b.

PITTENGER C, DUMAN RS: Stress, depression, and neuroplasticity: a convergence of mechanisms. Neuropsychopharmacology 33: 88-109, 2008.

REILLY JL, LENCER R, BISHOP JR, KEEDY S, SWEENEY JA: Pharmacological treatment effects on eye movement control. Brain Cogn 68: 415-435, 2008.

RIZZOLATTI G, LUPPINO G: The cortical motor system. Neuron 36: 155-165, 2001.

SHEPHERD M, FINDLAY JM, HOCKEY RJ: The relationship between eye movements and spatial attention. $Q J$ Exp Psychol A 38: 475-491, 1986.

SWEENEY JA, LUNA B, KEEDY SK, MCDOWELL JE, CLEMENTZ BA: fMRI studies of eye movement control: Investigating the interaction of cognitive and sensorimotor brain systems. Neuroimage 36: T54-T60, 2007.

THICKBROOM GW, MASTAGLIA FL: Presaccadic spike potentials. Relation to eye movement direction. Electroencephalogr Clin Neurophysiol 64: 211-214, 1986.

TRILLENBERG P, LENCER R, HEIDE W: Eye movements and psychiatric disease. Curr Opin Neurol 17: 43-47, 2004.

WARABI T, KASE M, KATO T: Effect of aging on the accuracy of visually guided saccadic eye movement. Ann Neurol 16: 449-454, 1984.

WONG B, CRONIN-GOLOMB A, NEARGARDER S: Patterns of visual scanning as predictors of emotion identification in normal aging. Neuropsychology 19: 739-749, 2005.

YARBUS AL: Eye Movements and Vision. Plenum Press, New York, 1967, 222 p. 